\title{
A Multi-Agent Design for a Home Automation System dedicated to power management
}

\author{
Shadi ABRAS ${ }^{1}$, Stéphane PLOIX ${ }^{2}$, Sylvie PESTY ${ }^{1}$, and Mireille \\ $\mathrm{JACOMINO}^{2}$ \\ ${ }^{1}$ Laboratoire LIG-Institut IMAG,CNRS, UMR5217, \\ ${ }^{2}$ Laboratoire G-SCOP, CNRS, UMR5528, \\ 46, Avenue Félix Viallet, 38031 Grenoble, France. \\ Phone: 00334765750 59, Fax: 0033476574602 \\ \{Shadi.Abras, Sylvie.Pesty\}@imag.fr \\ \{Stephane.Ploix, Mireille.Jacomino\}@inpg.fr
}

\begin{abstract}
This paper presents the principles of a Home Automation System dedicated to power management that adapts power consumption to available power ressources according to user comfort and cost criteria. The system relies on a multi-agent paradigm. Each agent, supporting a type of service achieved by one or several devices, cooperates and coordinates its action with others in order to find an acceptable near-optimal solution. The control algorithm is decomposed into two complementary mechanisms: a reactive mechanism, which protects from constraint violations, and an anticipation mechanism, which computes a predicted plan of global consumption according to predicted productions and consumptions and to user criteria. The paper presents a design for the Multi Agent Home Automation System.
\end{abstract}

\section{Introduction}

A building is both a place of power consumption and potentially a place of decentralized power production using resources like wind, solar, geothermal, etc. The resort to renewable power resources comes up in homes knowing that buildings represent $47 \%$ of the global power consumption [2]. Therefore, the design of a control system which allows the exploitation of different energy resources, while managing globally the power needs and the production capacity of a home, is an upcoming issue.

The role of a Home Automation System dedicated to power management is to adapt the power consumption to the available power resources, and vice verse, taking into account inhabitant comfort criteria. It has to reach a compromise between the priorities of inhabitants in terms of comfort and cost.

Algorithms based on MAS are nowadays used in several areas such as Computer Science or Automatic Control. The design of solutions based on Multi-Agent Systems, which are well suited to solve spatially distributed and open problems, facilitates the design of an intelligent Multi-Agent Home Automation System

Please use the following format when citing this chapter:

Abras, S., Ploix, S., Pesty, S., Jacomino, M., 2007, in IFIP International Federation for Information Processing, Volume 247, Artificial Intelligence and Innovations 2007: From Theory to Applications, eds. Boukis, C., Pnevmatikakis, L., Polymenakos, L., (Boston: Springer), pp. 233-241. 
(MAHAS). This paper presents the architecture of a MAHAS and shows why a MAS are particularly well suited for this class of problems.

\section{Multi-Agent Home Automation System}

A MAHAS consists of agents, each agent supports one type of service (heating, cooking, etc) achieved by one or several devices. The main features of the MAHAS are the following:

- Distributed: the energy resources and devices are independent and spatially distributed.

- Flexible: some devices can accumulate energy (different kinds of heating services) or satisfy with a timed delay the demands of inhabitants (washing service, etc).

- Open: the number of connected resources and devices may vary with time without having to completely redefine the control mechanism.

- Extendable: agents dedicated to new kinds of services may appear.

In MAS, the notion of control involves operations such as coordination and negotiation among agents, elimination of agents and addition of new agents when needed. Depending on weather forecasts, power resource information and inhabitant habits:

- an agent, dedicated to a power source, is assumed to be capable of calculating the future available power production.

- an agent, dedicated to a load, is assumed to be capable of calculating the prediction of power consumption: to determine what are the future power needs taking into account the usual behaviour of inhabitants.

In MAHAS, an additional agent is responsible for broadcasting the computed predicted plans to the other agents.

The structure of the MAHAS is decomposed into two main mechanisms: the reactive mechanism and the anticipation mechanism.

\subsection{Reactive mechanism}

The reactive mechanism is a short time adjustment mechanism which is triggered when the level of satisfaction of an agent falls below weak values ( $10 \%$ for example). This mechanism, which relies on the negotiation protocol [1], reacts quickly to avoid violations of energy constraints due to unpredicted perturbations and to guarantee a good level of inhabitant satisfaction. Therefore, the reactive mechanism adjusts, with a short sample time, the set points coming from the predicted plan, the device's current state (device satisfaction value) and the constraints and inhabitant criteria. 


\subsection{Anticipative mechanism}

The reactive mechanism is sufficient to avoid constraint violations but a MAHAS can be improved in order to avoid frequent emergency situations. This improvement is obtained due to the anticipation mechanism. The objective of this mechanism is to compute plans for production and consumption of services in a house. The anticipation mechanism benefits from, on the one hand, some devices are capable of accumulating energy, and on the other hand, some services have a variable date for their execution: some services can be either delayed or advanced. From these preliminary observations, it is possible to imagine that if the device consumption can be anticipated, there is a way to organize it better.

It works on a time window, which corresponds to a sampling period called the anticipative period. This period is greater than the one used by the reactive mechanism (typically 10 times). Because of the large sampling period, it considers average values of energy. This is an advantage when considering prediction because it is difficult to make precise predictions. The predicted set points can be directly transmitted to devices or adjusted by the reactive mechanism in case of constraint violation.

Because the reactive mechanism has been presented in detail in [1], this paper focuses on the anticipative mechanism.

\section{M.A.H.A.S modelling}

One of the objectives of the MAHAS is to fulfil inhabitant comfort, a notion which can be linked directly to the concept of satisfaction function [5]. Satisfaction functions have been defined for power resources as well as for devices. A satisfaction function is expressed by a function defined from the domain of characteristic variables to the interval $[0,1]$. In MAHAS, satisfaction characterizes the inhabitant's feeling about a service where zero means "unacceptable" and 1 means "fully satisfied". For example, satisfaction function for a thermal air environment is based on room temperature values (figure 1). For instance, figure 1 can be represented by the set $\{(T M I N$ (heating), 0$),(T P R E$ (heating), 1$)$, (TMAX (heating), 0$)\}$, it is implicitly assumed that before the first abscisse and after the last one, the satisfaction is null. A Home Automation System aims at

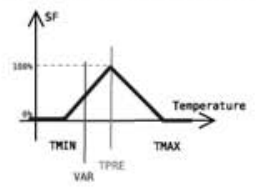

Fig. 1. Thermal air environment satisfaction function.

reaching a compromise between the inhabitant requests in terms of comfort and cost while satisfying technological constraints of devices. A MAHAS takes into 
account the price variation because the energy providers could charge inhabitants for the actual energy production cost in real time. Therefore, a MAHAS aims at minimizing the cost while maximizing the users comfort.

To optimize the criteria, the agents (figure 2) must communicate and cooperate in exchanging messages based on a common knowledge model. As mentioned

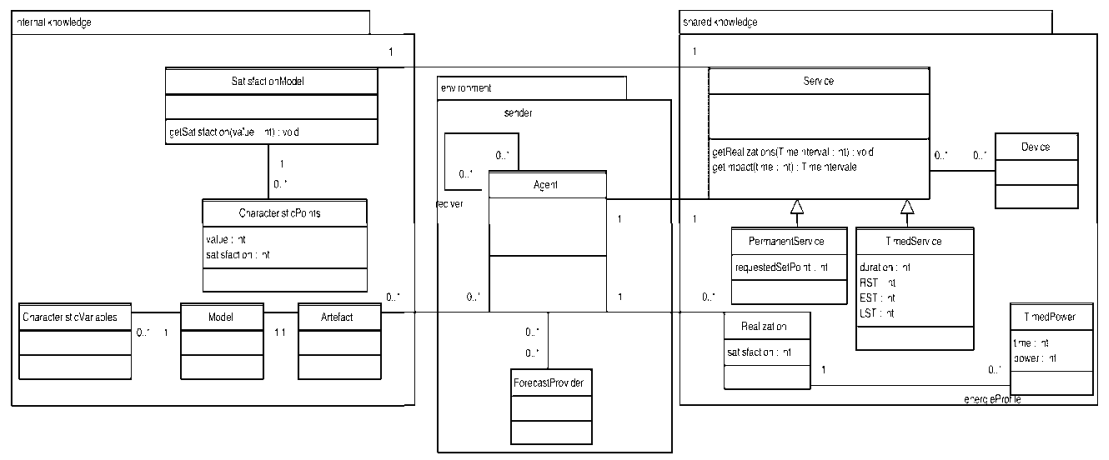

Fig. 2. MAHAS UML model.

in section 2, a MAHAS is "open" and "extendable" and because there are many kinds of services in a home, which provide predictions based on specific physical constraints, some knowledge are not shareable. It is indeed very difficult to formalize in a UML class diagram the structure of a general model, which can be composed of differential equations, recurrent equations, state machine, rules or a mix of all. It is all the more difficult that the system is extendable, it is not possible to circumscribe all the possible kinds of models. Just like for humans, some knowledge cannot be formalized in a general way. MAHAS is particularly suited because agents endowed into devices may embed their own knowledge and exchange messages based only on shareable knowledge which can be analysed by all the agents (for example: a power profile of a service represents a series of consumed or supplied powers corresponding to consecutive sampling periods). These shared data have to be formalized in a standardized form. In figure 2, an agent has its internal knowledge that gathers all the data that cannot be formalized in a general manner. Each agent embeds non-shareable piece of knowledge, build up its own representation of its environment, including other agents and interacts with other agents with a protocol [1] that deals with shareable knowledge.

\subsection{Agent modelling}

In the MAHAS, an agent supports a service $S R V_{i} \in \mathcal{S R V \mathcal { S }}$ where $\mathcal{S R V \mathcal { S }}$ is the set of all services. A service $S R V_{i}$ may be either a timed service, denoted $S R V_{i}^{T}$ , or a permanent service, denoted $S R V_{i}^{P}$, achieved by one or several devices, knowing that a device may also achieve many services. 
Modelling of a timed service agent A timed service corresponds to power profiles during a given period; the flexibility of the service comes from the possibility that it may shifted. The internal knowledge of a timed service agent is modeled by:

- a characteristic variable which corresponds to the starting time of the service.

- a behavioral model that defines the possible power profiles once the service has been started.

- a satisfaction function defined from the set of possible starting time to the set of possible satisfaction values. The parameters of the satisfaction function may be gathered in a set of characteristic points: $\left\{\left(E S T\left(S R V_{i}\right), 0\right),\left(R S T\left(S R V_{i}\right), 1\right),\left(L S T\left(S R V_{i}\right), 0\right)\right\}$ that represent respectively the earliest (unacceptable satisfaction), requested (fully satisfied) and latest starting times for the timed service.

A realization for a timed service agent represents the shared knowledge about a timed service. It is composed of:

- a power profile: $\Pi=\left[\operatorname{POW}\left(S R V_{i}\right)_{0}, \ldots, P O W\left(S R V_{i}\right)_{n}\right]$ where $\operatorname{POW}\left(S R V_{i}\right)_{j} \neq$ 0 represents the average power consumption/production (negative/positive) of the service during the $k^{\text {th }}$ sampling period. The duration of a service is determined by the length of the power profile length $(I)$.

- a satisfaction value which depends on the starting time of the service.

A realization corresponds to an instantiated power profile denoted: $R E A Z\left(S R V_{i}^{T}\right)=(k, \Pi, \sigma)$, where $k$ is the selected starting sampling period, $\Pi$ is the power profile and $\sigma$ is the satisfaction value modelling the inhabitant's feeling about this realization.

Modelling of a permanent service agent A permanent service corresponds to power profiles covering a given period. The flexibility of the service comes from the possibility of adjusting energy allocation from time to time. The internal knowledge of a permanent service agent is modeled by:

- a behavioral model linking the power profile to effect an inhabitant environment.

- a satisfaction function defined from a set of variables computed by the behavioral model to the set of possible satisfaction values. The parameters of the satisfaction function may be gathered in a set of characteristic points.

A realization for a permanent service agent represents the shared knowledge of a timed service. It is composed of:

- a power profile: $\Pi=\left[\ldots, P O W\left(S R V_{i}\right)_{k}, \ldots\right]$ where $P O W\left(S R V_{i}\right)_{k}$ represents the average power consumption/production during the $k^{\text {th }}$ period.

- a satisfaction value. Even if a realization covers several periods, there is only one value that models the inhabitant resulting satisfaction for the whole considered period: it is usually equal to the minimum of the satisfaction values computed for each period. 
A realization corresponds to an instantiated power profile denoted: $R E A Z\left(S R V_{i}^{P}\right)=(k, I, \sigma)$, where $\Pi$ is a power profile from the sampling period $[k, k+$ length $(\Pi)]$ and $\sigma$ is a satisfaction value modelling the inhabitant's feeling about this realization.

Let's define the notion of impact of a service for a given sampling period $k$. It corresponds to the set of realizations for a service $S R V_{i}$, which is affected by a change of power affectation at the given time $k$. For a permanent service, the impact coresponds to $[k-p, k+p]$ where $p \times \Delta$ corresponds to the time response of the related devices. For a timed service, it coresponds to the set of realizations defined at $k$.

Even if the methods for solving the optimization problem may provide good solutions, they still require long computation times and considerable working memory because the complexity is NP-Hard [4]. A relevant optimization strategy (section 4), exploiting the nature of the Home Automation System problem, is a promising avenue for performance improvement.

\section{Optimization strategy}

An intelligent power management problem in buildings can be divided into subproblems involving different agents because, generally, inhabitants do not use their devices most of the time. The basic principle is to divide the whole problem into independent sub-problems then to solve each sub-problem independently in order to find a solution for the whole problem. The advantage of this method is to reduce the complexity of the whole problem which depends on the number of periods for each sub-problem and on the number of devices. When the whole problem is divided into sub-preblems, each sub-problem does not involve all of the services (for example: some inhabitants vacuum clean in the morning and not in the evening). Because the number of considered periods in a sub-problem is lower than the number of periods of the whole problem [3], the complexity of solving the sub-problems is less than the complexity of solving the whole problem at once.

The principle of the division of the problem into sub-problems is described in the following procedure:

- procedure solving of the whole problem

- distribute the energy over all periods for all the services according to the inhabitant's satisfaction;

if insufficient energy for some periods then

detect sub-problems;

determine the time interval for each sub-problem;

solve each sub-problem separately;

merge the sub-problems solutions to obtain a global plan;

else a solution according to the inhabitant's satisfaction is selected;

end if;

- end procedure;

This procedure is described in the next sub-sections. 


\subsection{Energy distribution}

The search for the global solution starts by distributing the available energy over all the periods for all the services according to the inhabitant's fully satisfaction where agents provide realizations with a satisfaction value equal to 1 and where agents, dedicated to a power resource, provide one realization that is the best prediction over all periods. When agents has several realizations with a satisfaction equal to 1, the solving agent verifies, using the Bellman-Ford's algorithm, if the agent realizations are acceptable or not according to the available energy. At the end of the energy distribution, if there is enough energy for all devices, the search for a solution is stopped and a solution according to the inhabitant's fully satisfaction is obtained. But if there is not enough energy for all devices, sub-problems will be detected (sub-section 4.2) and then will be solved (sub-section 4.3).

\subsection{Determination of the sub-problem interval}

When the service agents suppling power have not enough power for all devices, the solving agent sends the periods for which there is not enough energy to all permanent and timed service agents. To obtain independent sub-problems, it is necessary to determine the service influences over theses periods. The principle of the sub-problem interval determination is described as follows:

First, compute the earliest and the latest starting time for the timed services:

- each realization of a timed service agent is characterized by a starting time, an ending time (equal to the starting time plus the length of the power profile).

- each timed service agent sends their intervals to the solving agent.

Each permanent service agent sends its impact to the solving agent.

The solving agent creates several intervals (sub-problem intervals) depending on the number of periods where there is not enough energy.

- The solving agent verifies the intersection between the sub-problem intervals and the earliest and the latest time of the timed services; it chooses the minimum value between the earliest time and the inferior bound of the interval and the maximum value between the latest time and the superior bound of the interval.

- The solving agent adds the impact of the permanent agent to the sub-problem intervals; the fact of adding the impact of permanent service agents increases the interval of the sub-problem but it guarantees that there is no influence on the previous or next periods.

- The solving agent merges two sub-problem intervals if there is an intersection between these two sub-problem intervals.

Then the solving agent sends the sub-problems intervals to all agents. 


\subsection{Resolution of a sub-problem}

The solving agent computes a predicted plan for each sub-problem using the Tabu Search (TS) algorithm [6]. The basic principles of TS for the MAHAS problem are the following: the search for a solution starts from the initial solution found at the energy distribution step. At each iteration, the solving agent decreases the satisfaction interval (for example $5 \%$ for each time) and sends it with the best realizations (according to a combination of cost and comfort criteria) at the previous iteration to the agents; agents computes the neighborhood of the realization sent by the solving agent by generating a given number of realizations corresponding to the satisfaction interval. When the solving agent receives the agent realizations, it chooses the realizations that violate the constraints the less as possible. The search is stopped when the collected realizations do not violate the global power constraints and when the global satisfaction has converged. Because the number of realizations corresponding to a satisfaction interval is very high, an agent generates randomly theses realizations corresponding to the satisfaction in performing elementary step from the realization selected by the solving agent at the previous iteration knowing that an agent realization will not be generated and sent twice to the solving agent.

\section{Conclusion}

This paper has presented the principles of a MAHAS which allows the agents to cooperate and coordinate their actions in order to find an acceptable nearoptimal solution for power management. A cooperation mechanism that reduces the problem complexity has been detailed. It has also been shown why autonomous and cooperative agent are particularly well suited for spatially distributed, flexible, open and extendable context such as power management in buildings, in pointing out that there is non shareable knowledge. This feature is a clear improvement over the capabilities provided by current Automatic Controlled Systems. A simulator has been designed and results let imagine a new way for producing and consuming power. The next step is the implementation in a real building.

\section{References}

1. Abras, S., Ploix, S., Pesty, S., and Jacomino, M. (2006). A multi-agent home automation system for power managememnt. In Proceedings of the Third International Conference in Control, Automation, and Robotics, ICINCO 2006, pages $3-8$, Setúbal, Portugal.

2. Fontaine, N. (2003). Livre blanc sur les énergies. débat national sur les énergies, http://www.industrie.gouv.fr/energie/politiqu/ploe.htm. 
3. Habbas, Z., M. Krajecki and D. Singer (2005). Decomposition techniques for parallel resolution of constraint satisfaction problems in shared memory: a comparative study. International Journal of Computational Science and Engineering (IJCSE) 1(2), 192-206.

4. Garey, M. R. and D. S. Johnson (1979). Computers and Intractability: A Guide to the Theory of NP-Completeness. W.H. Freeman and Co. New York, USA.

5. Lucidarme, P., O. Simonin and A. Liégeois (2002). Implementation and evaluation of a satisfaction/altruism based architecture for multi-robot systems. In: Proceedings of the 2002 IEEE International Conference on Robotics and Automation. Washington, USA. pp. 1007-1012.

6. N. Koji and I. Toshihide. A tabu search approach to the constraint satisfaction problem as a general problem solver. European Journal of Operational Research, 106:599-623, 1998. 